\title{
The Periodicity of Entire Functions with Finite Order
}

\author{
Zhiguo Ren ${ }^{1}$ and Guoqiang Dang $\mathbb{D}^{2}$ \\ ${ }^{1}$ Department of Education and Educational Technology, Jiangmen Polytechnic, Jiangmen 529090, Guangdong, China \\ ${ }^{2}$ School of Mathematical Sciences, Beijing Normal University, Beijing 100875, China
}

Correspondence should be addressed to Guoqiang Dang; dang@mail.bnu.edu.cn

Received 4 March 2021; Revised 6 April 2021; Accepted 9 April 2021; Published 6 May 2021

Academic Editor: kit C. Chan

Copyright (c) 2021 Zhiguo Ren and Guoqiang Dang. This is an open access article distributed under the Creative Commons Attribution License, which permits unrestricted use, distribution, and reproduction in any medium, provided the original work is properly cited.

\begin{abstract}
This paper is concerned with the periodicity of entire functions with finite growth order, and some sufficient conditions are given. Let $f$ is a transcendental entire function with finite growth order, zero is a Picard exceptional value of $f$, and a given differential monomial $Q(f)$ of $f$ is periodic, then $f$ is also periodic. We are also interested in finding the following: let $f$ is a transcendental entire function with finite growth order, $d$ is a Picard exceptional value of $f$ and $f(z) \Delta_{c}^{n} f(z)$ is a periodic function, then $f$ is also a periodic function. These results extend Yang's conjecture.
\end{abstract}

\section{Introduction}

The aim of this paper is to bring together and generalize recent research work by Wang and $\mathrm{Hu}$ [1], Liu and $\mathrm{Yu}$ [2], Deng and Yang [3], Liu et al. [4], Lü and Zhang [5], and Liu et al. [6] which is related to Yang's conjecture $[1,2]$.

Conjecture 1 (Yang's conjecture $[1,2]$ ). Let $f$ be a transcendental entire function and $k$ be a positive integer. If $f f^{(k)}$ is a periodic function, then $f$ is also a periodic function.

We assume that the readers are familiar with Nevanlinna's theory [7-9]. Denote by $S(r, f)$ any quantify satisfying $S(r, f)=o(T(r, f)), r \longrightarrow \infty$, outside of a possible exceptional set of finite logarithmic measure. For a meromorphic function $f(z)$, define its shift by $f_{c}(z)=f(z+c)$ and its difference operators by

$$
\begin{aligned}
& \triangle_{c} f(z)=f(z+c)-f(z), \\
& \triangle_{c}^{n} f(z)=\triangle_{c}^{n-1}\left(\triangle_{c} f(z)\right) .
\end{aligned}
$$

For a nonconstant meromorphic function $f(z)$, the order $\sigma$ of $f(z)$ is defined by

$$
\sigma=\sigma(f)=\limsup _{r \rightarrow \infty} \frac{\log T(r, f)}{\log r} .
$$

Wang and $\mathrm{Hu}[1]$ proved the following theorem.
Theorem 1 (see [1, Theorem 1]). Let $f$ be a transcendental entire function and let $k$ be a positive integer. If $\left(f^{2}\right)^{(k)}$ is a periodic function, then $f$ is also a periodic function.

If $f$ is a transcendental entire function with a nonzero Picard exceptional value, Liu and Yu [2] proved the following theorem in 2019.

Theorem 2 (see [2, Theorem 1.1]). Let $f$ be a transcendental entire function with a nonzero Picard exceptional value and let $k$ be a positive integer. If $f f^{(k)}$ is a periodic function, then $f$ is also a periodic function.

In fact, Theorem 1 shows that Yang's conjecture is true when $k=1$. The transcendental entire function $f^{2}$ cannot replaced by $f$ in Theorem 1 [2], due to a counterexample which had been presented by Liu and Yu which shows that $f(z)=e^{z}+z$ is not a periodic function, but $f^{(k)}(z)$ is a periodic function [2]. They also depicted that the function $f^{2}$ can be replaced by $f^{n}$ in Theorem 1 provided that $n \geq 3$ [2]. In Theorem 2, Liu and $\mathrm{Yu}$ [2, Theorem 1.1] investigated the periodicity of a transcendental entire function with a nonzero Picard exceptional. Furthermore, in Theorem 1, if we restrict $f$ be a transcendental entire function with finite order and zero is a Picard exceptional value, we can obtain the following more refined theorem which shows that $f$ has an explicit expression. 
Theorem 3. Let $f$ be a transcendental entire function with finite order, zero be a Picard exceptional value of $f$, and $k$ be a positive integer. If $\left(f^{2}\right)^{(k)}$ is a periodic function with a period of $c$, then $f$ is also a periodic function with a period of $2 c$; furthermore, $f(z)=e^{a z+b}$, where $a \neq 0, b$ are constants and $e^{2 a c}=1$.

If we replace $\left(f^{2}\right)^{(k)}$ by $f\left(f^{2}\right)^{(k)}$ in Theorem 3 , we can obtain the following theorem.

Theorem 4. Let $f$ be a transcendental entire function with finite order and zero be a Picard exceptional value of $f$, and let $k$ be a positive integer. If $f\left(f^{2}\right)^{(k)}$ is a periodic function with a period of $c$, then $f$ is also a periodic function with a period of $3 c$; furthermore, $f(z)=e^{a z+b}$, where $a \neq 0, b$ are constants and $e^{3 a c}=1$.

In 2020, under the conditions of Theorem 2, if the condition " $f$ with a nonzero Picard exceptional value" replaced by "zero be a Picard exceptional value of $f$," Deng and Yang [3] proved the following theorem.

Theorem 5 (see [3, Theorem 3]). Let $f$ be a transcendental entire function with finite order and zero be a Picard exceptional value of $f$ and let $k$ be a positive integer. If $f f^{(k)}$ is a periodic function with a periodic of $c$, then $f(z)$ is also a periodic function; furthermore, $f(z)=e^{a z+b}$, where $a(\neq 0), b$ are constants, $e^{2 a c}=1$, and $f(z)$ is with a period of $2 c$.

We generalize Theorem 5 and obtain the following result.

Theorem 6. Let $f$ be a transcendental entire function with finite order and zero be a Picard exceptional value of $f$ and let $k$ be a positive integer. If $f\left(f^{(k)}\right)^{2}$ is a periodic function with a period of $c$, then $f$ is also a periodic function with a period of $3 c$; furthermore, $f(z)=e^{a z+b}$, where $a \neq 0, b$ are constants and $e^{3 a c}=1$.

The following generalized Yang's conjecture has been considered by Liu et al. in 2020 [4].

Conjecture 2 (generalized Yang's conjecture [4]). Let $f$ be a transcendental entire function and $n, k$ be positive integers. If $f^{n} f^{(k)}$ is a periodic function, then $f$ is also a periodic function.

If $f$ is a transcendental entire function, Liu et al. [4] proved that the aforementioned generalized Yang's conjecture is true under the conditions that if $k=1$ or if $f(z)=e^{h(z)}$, where $h(z)$ is a nonconstant polynomial, or if $f(z)$ has a nonzero Picard exceptional value and $f(z)$ is of finite order. In 2021, Liu et al. [6] proved that the generalized Yang's conjecture is true for meromorphic functions in the case of $k=1$, while for $k \geq 2$, the conjecture is true under some certain conditions even if $n$ is a negative integer value. Baker [10] proved that if $f(z)$ is a nonconstant entire function and $p(z)$ is a polynomial with $\operatorname{deg}(p) \geq 3$, then $f(p)$ is not a periodic function. Contrarily, when $p(f)$ is periodic, where $p$ is a nonconstant polynomial, it is interesting to discuss the periodicity of $f$. Let $f$ be a transcendental entire function, $k$ be a positive integer, $a_{i}(1 \leq i \leq n)$ be constants, and $a_{n} \neq 0$; Liu and
$Y u$ [2, Theorem 1.5] proved that if $\left(a_{2} f^{2}+a_{1} f\right)^{(k)}(n \geq 2)$ is a periodic function, then $f$ is also a periodic function. Staring form Theorems 3-6, we have a question that whether $f$ is also periodic if we change the conditions $\left(f^{2}\right)^{(k)}, f\left(f^{2}\right)^{(k)}$, and $f\left(f^{(k)}\right)^{2}$ into a differential polynomial of $f(z)$. Furthermore, let $f$ is a transcendental entire function with $\sigma_{2}(f)<1$ and $n \geq 2, k$ are two positive integers, and $N(r, 1 / f)=S(r, f)$, Liu and $Y u$ ([2], Theorem 1.7) also proved that if $\left(a_{n} f^{n}+\cdots+a_{1} f\right)^{(k)}(n \geq 2)$ is a periodic function, $a_{n} \neq 0$, then $f$ is also a periodic function, but the exact formula of $f(z)$ is still unobtained [2]. In order to construct the explicit expression of $f$, it is necessary to put some restrictions of $f$. In fact, we obtain the following theorem.

Theorem 7. Let $f$ be a transcendental entire function with finite order and zero be a Picard exceptional value of $f$, and let $k$ be a positive integer. If $Q(f)=f^{n_{0}}\left(f^{\prime}\right)^{n_{1}}\left(f^{\prime \prime}\right)^{n_{2}} \ldots$ $\left(f^{(k)}\right)^{n_{k}}$ is a periodic function with a period of $c$, then $f$ is also periodic with a period of $\gamma c$; furthermore, $f(z)=e^{a z+b}$, where $a(\neq 0), b$ are constants, $e^{\gamma a c}=1$, and $\gamma=n_{0}+n_{1}+n_{2}+\cdots$ $+n_{k}$.

Noting that if we set $n_{0}=1, n_{1}=\cdots=n_{k-1}=0$ and $n_{k}=2$, Theorem 7 will reduce to Theorem 6 .

In 2020, Deng and Yang [3] studied the difference analogue of Yang's conjecture and obtained the following theorem.

Theorem 8 (see [3, Theorem 4]). Let $f$ be a transcendental entire function with finite order and $d$ be a Picard exceptional value of $f$, and $f(z) \Delta_{c} f(z)$ be a periodic function; then, $f$ is also a periodic function.

In Theorem 3, if we replace $\left(f^{2}\right)^{(k)}$ into $\Delta_{c}\left(f^{2}\right)$, we can obtain the following theorem.

Theorem 9. Let $f$ be a transcendental entire function with finite order and $d$ be a Picard exceptional value of $f$, and $\Delta_{c}\left(f^{2}\right)$ be a periodic function; then, $f$ is also a periodic function.

Theorems 8 and 9 partly show the internal relation of periodicity between a transcendental entire function and differences; in a more general setting, we can put the following theorem.

Theorem 10. Let $f$ be a transcendental entire function with finite order and $d$ be a Picard exceptional value of $f$, and $f(z) \Delta_{c}^{n} f(z)$ be a periodic function; then $f$ is also a periodic function.

\section{Lemma}

Lemma 1 (see [11]). Let $f$ be a transcendental entire function with finite order and $c$ be a nonzero constant; then, $T(r, f(z+c))=T(r, f)+S(r, f)$.

Lemma 2 (see [7]). Let $f_{j}(z)(j=1,2, \ldots, n)$ be meromorphic functions and $f_{j}(z)(1 \leq j \leq n-1)$ be nonconstant; $\sum_{k=1}^{n} f_{j}(z) \equiv 1$, and for any $k(1 \leq k \leq n)$, 


$$
\sum_{i=1}^{n} N\left(r, \frac{1}{f_{j}}\right)+(n-1) \sum_{i=1}^{n} \bar{N}\left(r, f_{j}\right)<(\lambda+o(1)) T\left(r, f_{k}\right), \quad r \longrightarrow \infty, r \notin E,
$$

where $E \subset(1, \infty)$ is a set of finite measure; then, $f_{n}(z) \equiv 1$.

\section{Proofs of Theorems}

\subsection{Proof of Theorem 3}

Proof. By Theorem 1, it follows easily that $f$ is periodic. We only need to show that $f=e^{a z+b}$, where $a \neq 0$ and $e^{2 a c}=1$.

Suppose that $f(z)=e^{P(z)}$, where $P(z)$ is a polynomial, $\operatorname{deg} P(z) \geq 1, T(r, P)=S(r, f)$. Differentiate $f^{2} k$ times, $\left(f^{2}\right)^{(k)}=\left(e^{2 P(z)}\right)^{(k)}=H(P(z)) e^{2 P(z)}$, where $H(P(z))$ is a differential polynomial of $P(z), H(P(z)) \equiv 0$. Otherwise, if $H(P(z)) \equiv 0$, then $\left(f^{2}\right)^{(k)} \equiv 0$; then, $f(z)$ is a polynomial, but it is a contradiction with $f(z)$ which is a transcendental entire function.

Since $\left(f^{2}\right)^{(k)}$ is a periodic function with a period of $c$, $\left(f^{2}(z)\right)^{(k)}=\left(f^{2}(z+c)\right)^{(k)}$; consequently,

$$
H(P(z)) e^{2 P(z)}=H(P(z+c)) e^{2 P(z+c)},
$$

and in this way,

$$
\frac{H(P(z+c))}{H(P(z))}=\frac{e^{2 P(z)}}{e^{2 P(z+c)}}=\frac{1}{e^{2 P(z+c)-2 P(z)}} .
$$

In the event of $\operatorname{deg} P(z) \geq 2$, then $\operatorname{deg}[P(z+c)-P(z)] \geq 1$. By Lemma 1, we have $T(r, H(P(z)))=S(r, f), T(r, H(P(z+c)))=S(r, f)$. But

$$
T\left(r, e^{2 P(z+c)-2 P(z)}\right)=T\left(r, \frac{H(P(z))}{H(P(z+c))}\right)=S\left(r, e^{2 P(z+c)-2 P(z)}\right),
$$

which is impossible. Therefore, $\operatorname{deg} P(z)=1$.

It follows that $P(z)=a z+b, a \neq 0$; then, $f(z)=e^{a z+b}$, $\left(f^{2}\right)^{(k)}=(2 a)^{k} e^{2 a z+2 b},(2 a)^{k} e^{2 a z+2 b}=(2 a)^{k} e^{2 a z+2 a c+2 b}$; consequently, $e^{2 a c}=1$. Hence, $f(z+2 c)=e^{P(z+2 c)}=e^{a(z+2 c)+b}=$ $e^{a z+b}=f(z)$.

This finishes the proof.

\subsection{Proof of Theorem 4}

Proof. According to the proof of Theorem 3, we have $\left(f^{2}\right)^{(k)}=\left(e^{2 P(z)}\right)^{(k)}=H(P(z)) e^{2 P(z)}$, where $H(P(z))$ is a differential polynomial of $P(z), \quad \operatorname{deg} P(z) \geq 1$, and $H(P(z)) \neq \equiv$. $c$, then

Because $f\left(f^{2}\right)^{(k)}$ is a periodic function with a period of

$$
f(z)\left(f^{2}(z)\right)^{(k)}=f(z+c)\left(f^{2}(z+c)\right)^{(k)} .
$$

It follows that

$$
H(P(z)) e^{3 P(z)}=H(P(z+c)) e^{3 P(z+c)},
$$

and accordingly,

$$
\frac{H(P(z+c))}{H(P(z))}=\frac{e^{3 P(z)}}{e^{3 P(z+c)}}=\frac{1}{e^{3 P(z+c)-3 P(z)}} .
$$

In the event of $\operatorname{deg} P(z) \geq 2, \operatorname{deg}[P(z+c)-P(z)] \geq 1$. By Lemma 1 , we have $T(r, H(P(z)))=S(r, f), T(r, H(P(z+$ c)) $)=S(r, f)$. Therefore,

$$
T\left(r, e^{3 P(z+c)-3 P(z)}\right)=T\left(r, \frac{H(P(z))}{H(P(z+c))}\right)=S\left(r, e^{3 P(z+c)-3 P(z)}\right),
$$

which is impossible. Hence, $\operatorname{deg} P(z)=1$.

Assuming that $P(z)=a z+b, a \neq 0$. Then, $f(z)=e^{a z+b}$, $\left(f^{2}\right)^{(k)}=(2 a)^{k} e^{2 a z+2 b}$, and $(2 a)^{k} e^{3 a z+3 b}=(2 a)^{k} e^{3 a z+3 a c+3 b}$; consequently, $e^{3 a c}=1$. Hence, $f(z+3 c)=e^{P(z+3 c)}=$ $e^{a(z+3 c)+b}=e^{a z+b}=f(z)$; then, $f(z)$ is a periodic function with a period of $3 c$.

This finishes the proof.

\subsection{Proof of Theorem 6}

Proof. Suppose that $f(z)=e^{P(z)}$, where $P(z)$ is a polynomial, and $\operatorname{deg} P(z) \geq 1$ and $T(r, P)=S(r, f)$. Then, $f^{(k)}=\left(e^{P(z)}\right)^{(k)}=H(P(z)) e^{P(z)}$, where $H(P(z))$ is a differential polynomial of $P(z)$ and $H(P(z)) \equiv 0$. Otherwise, if $H(P(z)) \equiv 0$, then $f^{(k)} \equiv 0$; therefore, $f(z)$ is a polynomial, which is contradicting with $f(z)$ which is a transcendental entire function with finite order.

Since $f\left(f^{(k)}\right)^{2}$ is a periodic function with a period of $c$, then

$$
f(z)\left(f^{(k)}\right)^{2}=f(z+c)\left(f^{(k)}(z+c)\right)^{2},
$$

and in this way,

$$
H^{2}(P(z)) e^{3 P(z)}=H^{2}(P(z+c)) e^{3 P(z+c)} .
$$

Taking the virtue of Lemma 1, we have $T(r, H(P(z)))=S(r, f), \quad T(r, H(P(z+c)))=S(r, f)$. Therefore,

$$
\frac{H^{2}(P(z+c))}{H^{2}(P(z))}=\frac{e^{3 P(z)}}{e^{3 P(z+c)}}=\frac{1}{e^{3 P(z+c)-3 P(z)}} .
$$

In the event of $\operatorname{deg} P(z) \geq 2, \operatorname{deg}[P(z+c)-P(z)] \geq 1$. But

$$
T\left(r, e^{3 P(z+c)-3 P(z)}\right)=T\left(r, \frac{H^{2}(P(z))}{H^{2}(P(z+c))}\right)=S\left(r, e^{3 P(z+c)-3 P(z)}\right),
$$

which is impossible. Hence, $\operatorname{deg} P(z)=1$.

Assuming that $P(z)=a z+b, a \neq 0$. So, $f(z)=e^{a z+b}$, $\left(f^{(k)}\right)^{2}=a^{2 k} e^{2 a z+2 b}$, and by (11), we have $a^{2 k} e^{3 a z+3 b}=a^{2 k} e^{3 a z+3 a c+3 b}$; therefore, $e^{3 a c}=1$. Hence, $f(z+3 c)=e^{P(z+3 c)}=e^{a(z+3 c)+b}=e^{a z+b}=f(z) ; \quad$ consequently, $f(z)$ is a periodic function with a period of $3 c$. 
This finishes the proof.

\subsection{Proof of Theorem 7}

Proof. Suppose that $f(z)=e^{P(z)}$, where $P(z)$ is a polynomial, and $\operatorname{deg} P(z) \geq 1$ and $T(r, P)=S(r, f)$. Differentiate $f k$ times; then $(f)^{(k)}=\left(e^{P(z)}\right)^{(k)}=H(P(z)) e^{P(z)}$, where $H(P(z))$ is a differential polynomial of $P(z)$ and $H(P(z)) \equiv 0$. Otherwise, if $H(P(z)) \equiv 0$, then $\left(f^{2}\right)^{(k)} \equiv 0$; then, $f(z)$ is a polynomial, but it is contradicting with $f(z)$ which is a transcendental entire function with finite order. Therefore,

$$
\begin{aligned}
Q(f)= & f^{n_{0}}\left(f^{\prime}\right)^{n_{1}}\left(f^{\prime \prime}\right)^{n_{2}} \ldots\left(f^{(k)}\right)^{n_{k}} \\
= & e^{n_{0} P(z)} H_{1}^{n_{1}}(P(z)) e^{n_{1} P(z)} H_{2}^{n_{2}} \\
& (P(z)) e^{n_{2} P(z)} \ldots H_{k}^{n_{k}}(P(z)) e^{n_{k} P(z)} \\
= & H_{0}(P(z)) e^{\left(n_{0}+n_{1}+\cdots+n_{k}\right) P(z)} \\
= & H_{0}(P(z)) e^{\gamma P(z)},
\end{aligned}
$$

where $H_{0}(P(z))$ is a differential polynomial of $P(z)$. Since $Q(f)$ is a periodic function with a period of $c$, then $Q(f(z))=Q(f(z+c))$, and

$$
H_{0}(P(z)) e^{\gamma P(z)}=H_{0}(P(z+c)) e^{\gamma P(z+c)} .
$$

Therefore,

$$
\frac{H_{0}(P(z+c))}{H_{0}(P(z))}=\frac{e^{\gamma P(z)}}{e^{\gamma P(z+c)}}=\frac{1}{e^{\gamma P(z+c)-\gamma P(z)}} .
$$

In the event of $\operatorname{deg} P(z) \geq 2, \operatorname{deg}[P(z+c)-P(z)] \geq 1$. By Lemma 1 , we have $T\left(r, H_{0}(P(z))\right)=S(r, Q(f))$, $T\left(r, H_{0}(P(z+c))\right)=S(r, Q(f))$. But

$$
\begin{aligned}
T\left(r, e^{\gamma P(z+c)-\gamma P(z)}\right) & =T\left(r, \frac{H_{0}(P(z))}{H_{0}(P(z+c))}\right) \\
& =S\left(r, e^{\gamma P(z+c)-\lambda P(z)}\right),
\end{aligned}
$$

which is impossible. Hence, $\operatorname{deg} P(z)=1$.

Assuming that $P(z)=a z+b, a \neq 0$. So, $f(z)=e^{a z+b}$, $\left(f^{(k)}\right)^{n_{k}}=a^{k n_{k}} e^{n_{k} a z+n_{k} b}$, and by (16), we have $a^{\Gamma} e^{\gamma(a z+b)}=$ $a^{\Gamma} e^{\gamma(a z+a c+b)}$, where $\Gamma=n_{1}+2 n_{2}+3 n_{3}+\cdots+k n_{k}$, so $e^{\gamma a c}=1$. Therefore, $f(z+\gamma c)=e^{P(z+\gamma c)}=e^{a(z+\gamma c)+b}=e^{a z+b}=f(z)$, and hence $f(z)$ is a periodic function with a period of $\gamma c$.

This finishes the proof.

\subsection{Proof of Theorem 9}

Proof. Let $f(z)$ be a transcendental entire function with finite order and $d$ be a Picard exceptional value of $f(z)$; then, $f(z)=d+e^{P(z)}$, where $P(z)$ is a polynomial of $\operatorname{deg} P(z) \geq 1$, and $T(r, P)=S(r, f)$. By simple computation, we have

$$
\begin{aligned}
\Delta_{c} f^{2} & =e^{2 P(z+c)}-e^{2 P(z)}+2 d\left(e^{P(z+c)}-e^{P(z)}\right) \\
& =\left(e^{P(z+c)}-e^{P(z)}\right)\left(e^{P(z+c)}+e^{P(z)}+2 d\right) .
\end{aligned}
$$

(i) Case 1. If $d \neq 0$.

Case 1.1. If $\Delta_{c} f^{2}(z) \equiv 0$, then $e^{P(z+c)}-e^{P(z)}=0$; therefore, $f$ is a periodic function with a period of $c$.

Case 1.2. If $\Delta_{c} f^{2}(z) \equiv 0$. Assuming that $\lambda$ is a period of $\Delta_{c} f^{2}(z)$, then

$$
\Delta_{c} f^{2}(z+\lambda)=\Delta_{c} f^{2}(z)
$$

Case 1.2.1. $\lambda+c \neq 0$.

By $f(z)=d+e^{p(z)}$ and (20), we have

$$
\begin{aligned}
& e^{2 P(z+\lambda+c)}-e^{2 P(z+\lambda)}+2 d\left(e^{P(z+\lambda+c)}-e^{P(z+\lambda)}\right) \\
& =e^{2 P(z+c)}-e^{2 P(z)}+2 d\left(e^{P(z+c)}-e^{P(z)}\right) .
\end{aligned}
$$

Consequently,

$$
\begin{aligned}
& e^{2 P(z+\lambda+c)}-e^{2 P(z+c)}-e^{2 P(z+\lambda)}+e^{2 P(z)} \\
& \quad+2 d\left(e^{P(z+\lambda+c)}-e^{P(z+\lambda)}-e^{P(z+c)}+e^{P(z)}\right)=0 .
\end{aligned}
$$

Furthermore,

$$
\begin{aligned}
& e^{2 P(z)}\left(e^{2 P(z+\lambda+c)-2 P(z)}-e^{2 P(z+c)-2 P(z)}-e^{2 P(z+\lambda)-2 P(z)}+1\right) \\
& \quad+2 d e^{P(z)}\left(e^{P(z+\lambda+c)-P(z)}-e^{P(z+\lambda)-P(z)}-e^{P(z+c)-P(z)}+1\right)=0 .
\end{aligned}
$$

Therefore, we must have

$$
\begin{gathered}
e^{2 P(z)}\left(e^{2 P(z+\lambda+c)-2 P(z)}-e^{2 P(z+c)-2 P(z)}-e^{2 P(z+\lambda)-2 P(z)}+1\right)=0, \\
2 d e^{P(z)}\left(e^{P(z+\lambda+c)-P(z)}-e^{P(z+\lambda)-P(z)}-e^{P(z+c)-P(z)}+1\right)=0 .
\end{gathered}
$$

It follows that

$$
e^{2 P(z+\lambda+c)-2 P(z)}-e^{2 P(z+c)-2 P(z)}-e^{2 P(z+\lambda)-2 P(z)}+1=0,
$$

$$
2 d\left(e^{P(z+\lambda+c)-P(z)}-e^{P(z+\lambda)-P(z)}-e^{P(z+c)-P(z)}+1\right)=0 .
$$

If not, by Lemmas 1 and 2, combining (25) and (26), we have 


$$
T\left(r, e^{P(z)}\right)=T\left(r, \frac{2 d\left(-e^{P(z+\lambda+c)-P(z)}+e^{P(z+\lambda)-P(z)}-1+e^{P(z+c)-P(z)}\right)}{-e^{2 P(z+\lambda+c)-2 P(z)}+e^{2 P(z+c)-2 P(z)}-1+e^{2 P(z+\lambda)-2 P(z)}}\right)=S\left(r, e^{P(z)}\right)
$$

which is impossible.

Case 1.2.1.1. $\operatorname{deg} P \geq 2$.

Assuming that $P(z)=a_{n} z^{n}+a_{n-1} z^{n-1}+\cdots+$ $a_{0}, a_{n} \neq 0, n \geq 2$. By computation, we have $\Delta_{c} P(z)=$ $n a_{n} c z^{n-1}+p_{0}(z)$. Hence, $\quad P(z+\lambda+c)-P(z)$, $P(z+\lambda)-P(z), P(z+c)-P(z)$ are polynomials with degree no more than $n-1$; by (25) and Lemma 2 , we have $-e^{2 P(z+\lambda+c)-2 P(z)} \equiv 1$, or $e^{2 P(z+c)-2 P(z)} \equiv 1$, or $e^{2 P(z+\lambda)-2 P(z)} \equiv 1$, but it is impossible.

Case 1.2.1.2. $\operatorname{deg} P=1$.

By $P(z)=a_{1} z+a_{0}, \Delta_{c} P(z)=a_{1} c$ and (25), we have $e^{a_{1} z+a_{0}}\left(e^{2 a_{1}(\lambda+c)}-e^{2 a_{1} c}-e^{2 a_{1} \lambda}+1\right)=0$. By Lemma 2 , we have $e^{2 a_{1} \lambda} \equiv 1$, or $e^{2 a_{1} c} \equiv 1$, or $-e^{2 a_{1}(\lambda+c)} \equiv 1$; therefore, $f(z)$ is a function of a period of $2 \lambda$, or $2 c$, or $2(\lambda+c) \pi i$. Since $d \neq 0$, by $(26), \quad e^{a_{1}(\lambda+c)}-$ $e^{a_{1} \lambda}-e^{a_{1} c}+1=0$; furthermore $e^{a_{1} \lambda} \equiv 1$, or $e^{a_{1} c} \equiv 1$, or $-e^{a_{1}(\lambda+c)} \equiv 1$, and hence $f(z)$ is a periodic function with a period of $\lambda$ or $c$ or $(\lambda+c) \pi i$.

Case 1.2.2. $\lambda+c=0$.

By $f(z)=d+e^{p(z)}$ and (20), we have

$$
\begin{aligned}
& e^{2 P(z)}-e^{2 P(z-c)}+2 d\left(e^{P(z)}-e^{P(z-c)}\right) \\
& =e^{2 P(z+c)}-e^{2 P(z)}+2 d\left(e^{P(z+c)}-e^{P(z)}\right) .
\end{aligned}
$$

Consequently,

$$
2 e^{2 P(z)}-e^{2 P(z+c)}-e^{2 P(z-c)}+2 d\left(2 e^{P(z)}-e^{P(z-c)}-e^{P(z+c)}\right)=0 .
$$

Therefore, we must have

$$
\begin{aligned}
& 2 e^{2 P(z)}-e^{2 P(z+c)}-e^{2 P(z-c)}=0, \\
& 2 d\left(2 e^{P(z)}-e^{P(z-c)}-e^{P(z+c)}\right)=0 .
\end{aligned}
$$

It follows that

$$
\begin{gathered}
e^{2 P(z)}\left(2-e^{2 P(z+c)-2 P(z)}-e^{2 P(z-c)-2 P(z)}\right)=0, \\
2 d e^{P(z)}\left(2-e^{P(z-c)-P(z)}-e^{P(z+c)-P(z)}\right)=0 .
\end{gathered}
$$

If not, by Lemmas 1 and 2, combining (31) and (32), we have

$$
T\left(r, e^{P(z)}\right)=T\left(r, \frac{1-(1 / 2) e^{P(z-c)-P(z)}-(1 / 2) e^{P(z+c)-P(z)}}{1-(1 / 2) e^{2 P(z+c)-2 P(z)}-(1 / 2) e^{2 P(z-c)-2 P(z)}}\right)=S\left(r, e^{P(z)}\right),
$$

which is impossible.

Case 1.2.2.1. $\operatorname{deg} P \geq 2$.

Assuming that $P(z)=a_{n} z^{n}+a_{n-1} z^{n-1}+\cdots$ $+a_{0}, a_{n} \neq 0, n \geq 2$. By computation, we have $\Delta_{c} P(z)=n a_{n} c z^{n-1}+p_{0}(z)$. Hence, $P(z-c)-P(z)$, $P(z+c)-P(z)$ are polynomials with degree no more than $n-1$; by (31) and Lemma 2 , we have $e^{2 P(z+c)-2 P(z)} \equiv 2$, or $e^{2 P(z-c)-2 P(z)} \equiv 2$, but it is impossible.

Case 1.2.2.2. $\operatorname{deg} P=1$.

By $P(z)=a_{1} z+a_{0}, \Delta_{c} P(z)=a_{1} c$ and (31), we have $e^{2 a_{1} z+2 a_{0}}\left(2-e^{2 a_{1} c}-e^{-2 a_{1} c}\right)=0$. By Lemma 2 , we have $e^{-2 a_{1} c} \equiv 2$, or $e^{2 a_{1} c} \equiv 2$; therefore, $f(z)$ is a function of a period of $2(\ln (1 / 2)) c$. If $d \neq 0$, by (32), we have $2-e^{P(z-c)-P(z)}-e^{P(z+c)-P(z)}=0$, and it follows that $(1 / 2) e^{-a_{1} c} \equiv 1$, or $(1 / 2) e^{a_{1} c} \equiv 1$, and hence $f(z)$ is a periodic function with a period of $(\ln (1 / 2)) c$.

(ii) Case 2. If $d=0$.

Case 2.1. If $\Delta_{c} f^{2}(z) \equiv 0$, then $e^{2 P(z+c)}-e^{2 P(z)}=0$; therefore, $f$ is a periodic function with a period of $c$.

Case 2.2. If $\Delta_{c} f^{2}(z) \equiv 0$. Assuming that $\lambda$ is a period of $\Delta_{c} f^{2}(z)$, then

$$
\Delta_{c} f^{2}(z+\lambda)=\Delta_{c} f^{2}(z) .
$$

Case 2.2.1. $\lambda+c \neq 0$.

By $f(z)=e^{p(z)}$ and (34), we have

$$
e^{2 P(z+\lambda+c)}-e^{2 P(z+\lambda)}=e^{2 P(z+c)}-e^{2 P(z)} .
$$

Consequently, 
$e^{2 P(z+\lambda+c)}-e^{2 P(z+c)}-e^{2 P(z+\lambda)}+e^{2 P(z)}=0$.

Furthermore,

$e^{2 P(z)}\left(e^{2 P(z+\lambda+c)-2 P(z)}-e^{2 P(z+c)-2 P(z)}-e^{2 P(z+\lambda)-2 P(z)}+1\right)=0$.

Therefore, we must have

$e^{2 P(z+\lambda+c)-2 P(z)}-e^{2 P(z+c)-2 P(z)}-e^{2 P(z+\lambda)-2 P(z)}+1=0$.

Case 2.2.1.1. $\operatorname{deg} P \geq 2$.

Assuming that $P(z)=a_{n} z^{n}+a_{n-1} z^{n-1}+\cdots+$ $a_{0}, a_{n} \neq 0, n \geq 2$. By computation, we have $\Delta_{c} P(z)=n a_{n} c z^{n-1}+p_{0}(z)$. Hence, $P(z+\lambda+c)-$ $P(z), P(z+\lambda)-P(z), P(z+c)-P(z)$ are polynomials with degree no more than $n-1$; by (38) and Lemma 2, we have $-e^{2 P(z+\lambda+c)-2 P(z)} \equiv 1$, or $e^{2 P(z+c)-2 P(z)} \equiv 1, \quad$ or $e^{2 P(z+\lambda)-2 P(z)} \equiv 1$, but it is impossible.

Case 2.2.1.2. $\operatorname{deg} P=1$.

By $P(z)=a_{1} z+a_{0}, \Delta_{c} P(z)=a_{1} c$ and (38), we have $e^{2 a_{1}(\lambda+c)}-e^{2 a_{1} c}-e^{2 a_{1} \lambda}+1=0$. By Lemma 2 , we have $e^{2 a_{1} \lambda} \equiv 1$, or $e^{2 a_{1} c} \equiv 1$, or $-e^{2 a_{1}(\lambda+c)} \equiv 1$; therefore, $f(z)$ is a function of a period of $2 \lambda$, or $2 c$, or $2(\lambda+c) \pi i$.

Case 2.2.2. $\lambda+c=0$.

By $f(z)=e^{p(z)}$ and (34), we have

$$
e^{2 P(z)}-e^{2 P(z-c)}=e^{2 P(z+c)}-e^{2 P(z)} .
$$

Consequently,

$$
2 e^{2 P(z)}-e^{2 P(z+c)}-e^{2 P(z-c)}=0 .
$$

It follows that

$$
e^{2 P(z)}\left(2-e^{2 P(z+c)-2 P(z)}-e^{2 P(z-c)-2 P(z)}\right)=0 .
$$

Case 2.2.2.1. $\operatorname{deg} P \geq 2$.

Assuming that $P(z)=a_{n} z^{n}+a_{n-1} z^{n-1}+\cdots+$ $a_{0}, a_{n} \neq 0, n \geq 2$. By computation, we have $\Delta_{c} P(z)=n a_{n} c z^{n-1}+p_{0}(z)$. Hence, $P(z-c)-P(z)$, $P(z+c)-P(z)$ are polynomials with degree no more than $n-1$; by (41) and Lemma 2, we have $e^{2 P(z+c)-2 P(z)} \equiv 2$, or $e^{2 P(z-c)-2 P(z)} \equiv 2$, but it is impossible.

Case 2.2.2.2. $\operatorname{deg} P=1$.

By $P(z)=a_{1} z+a_{0}, \Delta_{c} P(z)=a_{1} c$ and (41), we have $e^{2 a_{1} z+2 a_{0}}\left(2-e^{2 a_{1} c}-e^{-2 a_{1} c}\right)=0$. By Lemma 2 , we have $e^{-2 a_{1} c} \equiv 2$, or $e^{2 a_{1} c} \equiv 2$; therefore, $f(z)$ is a function of a period of $2(\ln (1 / 2)) c$.
This finishes the proof.

\subsection{Proof of Theorem 10}

Proof. $f(z)$ is a transcendental entire function with finite order and $d$ is its Picard exceptional value; then, $f(z)=d+e^{P(z)}$, where $P(z)$ is a polynomial of $\operatorname{deg} P(z) \geq 1$, and $T(r, P)=S(r, f)$. Then,

$$
\Delta_{c}^{n} f(z)=e^{P(z)} \sum_{j=0}^{n-1}(-1)^{j} C_{n}^{j} e^{P(z+(n-j) c)-P(z)}+(-1)^{n} e^{P(z)} .
$$

(i) Case 1. If $\Delta_{c}^{n} f(z) \equiv 0$.

We assert that $f(z)$ is a periodic function with a period of $c$. If $\operatorname{deg} P$ is odd, by (15), we have

$$
\sum_{j=0}^{n-1}(-1)^{j} C_{n}^{j} e^{P(z+(n-j) c)-P(z)}-1=0
$$

By Lemma 2, for each $j(0 \leq j \leq n-1)$, we have $(-1)^{j} C_{n}^{j} e^{P(z+(n-j) c)-P(z)} \equiv 1$. Therefore, $P(z+(n-$ j)c) $-P(z)=0$; then, $\quad f(z)=d+e^{P(z)}=d+$ $e^{P(z+(n-j) c)}=f(z+(n-j) c)$, and hence $f(z)$ is a periodic function with a period of $c$. If $\operatorname{deg} P$ is even, it is easy to prove that $f$ is also periodic.

(ii) Case 2. If $\Delta_{c}^{n} f(z) \equiv 0$.

Assuming that $\lambda$ is a periodic of $f(z) \Delta_{c}^{n} f(z)$, then

$$
f(z) \Delta_{c}^{n} f(z)=f(z+\lambda) \Delta_{c}^{n} f(z+\lambda) .
$$

Case 2.1. $\lambda+c \neq 0$.

By $f(z)=d+e^{P(z)}$ and (15) and (16),

$$
\begin{aligned}
& f(z)\left[e^{P(z)} \sum_{j=0}^{n-1}(-1)^{j} C_{n}^{j} e^{P(z+(n-j) c)-P(z)}+(-1)^{n} e^{P(z)}\right] \\
& =f(z+\lambda)\left[e^{P(z+\lambda)} \sum_{j=0}^{n-1}(-1)^{j} C_{n}^{j} e^{P(z+\lambda+(n-j) c)-P(z+\lambda)}+(-1)^{n} e^{P(z+\lambda)}\right] .
\end{aligned}
$$

Furthermore,

$$
\begin{aligned}
& \left(d+e^{P(z)}\right)\left[e^{P(z)} \sum_{j=0}^{n-1}(-1)^{j} C_{n}^{j} e^{P(z+(n-j) c)-P(z)}+(-1)^{n} e^{P(z)}\right] \\
& =\left(d+e^{P(z+\lambda)}\right)\left[e^{P(z+\lambda)} \sum_{j=0}^{n-1}(-1)^{j} C_{n}^{j} e^{P(z+\lambda+(n-j) c)-P(z+\lambda)}+(-1)^{n} e^{P(z+\lambda)}\right] .
\end{aligned}
$$

It follows that 


$$
\begin{aligned}
& d e^{P(z)}\left[\left(\sum_{j=0}^{n-1}(-1)^{j} C_{n}^{j} e^{P(z+(n-j) c)-P(z)}+(-1)^{n}\right)\right. \\
& \left.-e^{P(z+\lambda)-P(z)}\left(\sum_{j=0}^{n-1}(-1)^{j} C_{n}^{j} e^{P(z+\lambda+(n-j) c)-P(z+\lambda)}+(-1)^{n}\right)\right] \\
& +e^{2 P(z)}\left[\left(\sum_{j=0}^{n-1}(-1)^{j} C_{n}^{j} e^{P(z+(n-j) c)-P(z)}+(-1)^{n}\right)\right. \\
& \left.-e^{2 P(z+\lambda)-2 P(z)}\left(\sum_{j=0}^{n-1}(-1)^{j} C_{n}^{j} e^{P(z+\lambda+(n-j) c)-P(z+\lambda)}+(-1)^{n}\right)\right]=0 .
\end{aligned}
$$

Consequently,

$$
\begin{aligned}
& d\left[\left(\sum_{j=0}^{n-1}(-1)^{j} C_{n}^{j} e^{\Delta_{(n-j) c} P}+(-1)^{n}\right)-e^{\Delta_{\lambda} P}\left(\sum_{j=0}^{n-1}(-1)^{j} C_{n}^{j} e^{\Delta_{\lambda+(n-j) c} P}+(-1)^{n}\right)\right] e^{P(z)} \\
& \quad+\left[\left(\sum_{j=0}^{n-1}(-1)^{j} C_{n}^{j} e^{\Delta_{(n-j) c} P}+(-1)^{n}\right)-e^{2 \Delta_{\lambda} P}\left(\sum_{j=0}^{n-1}(-1)^{j} C_{n}^{j} e^{\Delta_{\lambda+(n-j) c} P}+(-1)^{n}\right)\right] e^{2 P(z)} \\
& =0 .
\end{aligned}
$$

Let

$$
\begin{array}{r}
A:=d\left[\left(\sum_{j=0}^{n-1}(-1)^{j} C_{n}^{j} e^{\Delta_{(n-j) c} P}+(-1)^{n}\right)-e^{\Delta_{\lambda} P}\left(\sum_{j=0}^{n-1}(-1)^{j} C_{n}^{j} e^{\Delta_{\lambda+(n-j) c} P}+(-1)^{n}\right)\right], \\
B:=\left[\sum_{j=0}^{n-1}(-1)^{j} C_{n}^{j} e^{\Delta_{(n-j) c} P}+(-1)^{n}\right]-e^{2 \Delta_{\lambda} P}\left[\sum_{j=0}^{n-1}(-1)^{j} C_{n}^{j} e^{\Delta_{\lambda+(n-j) c} P}+(-1)^{n}\right] .
\end{array}
$$

Hence, $A=0, B=0$. Otherwise, by Lemmas 1 and 2, $\operatorname{deg} \Delta_{(n-j) c} P<\operatorname{deg} P, \operatorname{deg} \Delta_{\lambda+(n-j) c} P<\operatorname{deg} P$, so $T\left(r, e^{P}\right)=$ $T(r,(A / B))=S\left(r, e^{P}\right)$, but it is impossible.

Case 2.1.1. $\operatorname{deg} P \geq 2$.

By simple computation, we have $\Delta_{c} P(z)=$ $m a_{m} c z^{m-1}+P_{0}(z)$. Furthermore,

$$
\begin{aligned}
\Delta_{(n-j) c} P(z) & =m a_{m}(n-j) c z^{m-1}+P_{1}(z), \\
\Delta_{\lambda+(n-j) c} P(z) & =m a_{m}(\lambda+(n-j) c) z^{m-1}+P_{2}(z),
\end{aligned}
$$

$P_{0}(z), P_{1}(z), P_{2}(z)$ are polynomials of degree no more than $m-2$; furthermore, $\Delta_{\lambda} P(z), \Delta_{(n-j) c} P(z)$, $\Delta_{\lambda+(n-j) c} P(z)$ are polynomials with degree of $m-1$, where $j=0, \ldots, n-1$; by $B=0$ and Lemma 2.2 , we have $(-1)^{j} C_{n}^{j} e^{\Delta_{(n-j) c} P}+(-1)^{n} \equiv 0$, or $(-1)^{n}-e^{2 \Delta_{\lambda} P}$ $(-1)^{n} \equiv 0$, or $-e^{2 \Delta_{\lambda} P}(-1)^{j} C_{n}^{j} e^{\Delta_{\lambda+(n-j) c} P}+(-1)^{n} \equiv 0$, but it is impossible.
Case 2.1.2. $\operatorname{deg} P=1$.

By $P(z)=a_{1} z+a_{0}, \Delta_{c} P(z)=a_{1} c, \Delta_{(n-j) c} P(z)=$ $a_{1}(n-j) c, \quad \Delta_{\lambda+(n-j) c} P(z)=a_{1}(\lambda+(n-j) c), \quad$ on account of $B=0$, we have

$$
\begin{aligned}
& \sum_{j=0}^{n-1}(-1)^{j} C_{n}^{j} e^{a_{1}(n-j) c}+(-1)^{n}-e^{2 a_{1} \lambda}\left(\sum_{j=0}^{n-1}(-1)^{j} C_{n}^{j} e^{a_{1}(\lambda+(n-j) c)}\right) \\
& \quad-(-1)^{n} e^{2 a_{1} \lambda}=0 .
\end{aligned}
$$

By Lemma 2 and $B=0$, when $n$ is even, we have $(-1)^{j} C_{n}^{j} e^{a_{1}(n-j) c}+1 \equiv 0, \quad$ or $(-1)^{j} C_{n}^{j} e^{a_{1}(\lambda+(n-j) c)+2 a_{1} \lambda}$ $\equiv 1, j=0, \ldots, n-1$, or $e^{2 a_{1} \lambda} \equiv 1$. Therefore, $f(z)$ is a periodic function with a period of $2 \lambda$ or $(n-j) c \ln \left((-1)^{j+1} C_{n}^{j}\right)$ or $(3 \lambda+(n-j) c) \ln \left((-1)^{j} C_{n}^{j}\right)$. If $d \neq 0$, by $A=0$, then $(-1)^{j} C_{n}^{j} e^{a_{1}(n-j) c}+1 \equiv 0$, or $e^{a_{1} \lambda} \equiv 1$, or $(-1)^{j} C_{n}^{j} e^{a_{1}(\lambda+(n-j) c)+a_{1} \lambda} \equiv 1, j=0, \ldots, n-1$, 
and hence $f(z)$ is a periodic function with a period of $\lambda$ or $(n-j) c \ln \left((-1)^{j+1} C_{n}^{j}\right)$ or $(2 \lambda+(n-j) c) \ln \left((-1)^{j}\right.$ $\left.C_{n}^{j}\right)$. The same proof for $n$ is odd. Therefore, $f(z)$ is a periodic function.

Case 2.2. $\lambda+c=0$.

This proof follows by the same method as in Case 2.1; just replace $\lambda$ by $-c$, so we omit it here.

This finishes the proof.

\section{Data Availability}

No data were used to support this study.

\section{Conflicts of Interest}

The authors declare that they have no conflicts of interest.

\section{References}

[1] Q. Wang and P. C. Hu, "On zeros and periodicity of entire functions," Acta Mathematica Scientia, Series A, vol. 38, no. 2, pp. 209-214, 2018.

[2] K. Liu and P. Yu, "A note on the periodicity of entire functions," Bulletin of the Australian Mathematical Society, vol. 100, no. 2, pp. 290-296, 2019.

[3] B. Deng and S. Yang, "Periodicity of entire functions concerning difference," Pure Mathematics, vol. 10, no. 2, pp. 96-99, 2020.

[4] K. Liu, Y. Wei, and P. Yu, "Generalized Yang's conjecture on the periodicity of entire functions," Bull. Korean Math. Soc.vol. 57, no. 5, pp. 1259-1267, 2020.

[5] W. Lü and X. Zhang, "On the periodicity of entire functions," Results in Mathematics, vol. 75, no. 4, 2020.

[6] X. Liu, R. Korhonen, and K. Liu, "Variations on a conjecture of C. C. Yang concerning periodicity," Computational Methods and Function Theory, vol. 59, 2021.

[7] C. C. Yang and H. X. Yi, Uniqueness Theory of Meromorphic Functions, Kluwer Academic Publishers, Berlin, Germany, 2003.

[8] I. Laine, Nevanlinna Theory and Complex Differential Equations, De Gruyter, Berlin, Germany, 2011.

[9] N. Steinmetz, Nevanlinna Theory, Normal Families, and Algebraic Differential Equations, Springer Cham, Berlin, Germany, 2017.

[10] I. N. Baker, "On some results of A. Rényi and C. Rényi concerning periodic entire functions," Acta Scientiarum Mathematicarum (Szeged), vol. 10, no. 27, pp. 197-200, 1966.

[11] Y.-M. Chiang and S.-J. Feng, "On the Nevanlinna characteristic of $\mathrm{f}(\mathrm{z}+\eta)$ and difference equations in the complex plane," The Ramanujan Journal, vol. 16, no. 1, pp. 105-129, 2008. 